\title{
Towards Efficient Sensor Placement for Industrial Wireless Sensor Network
}

\author{
R. Pavithra, D. Arivudainambi \\ Department of Mathematics, Anna University, Chennai - 600025, India
}

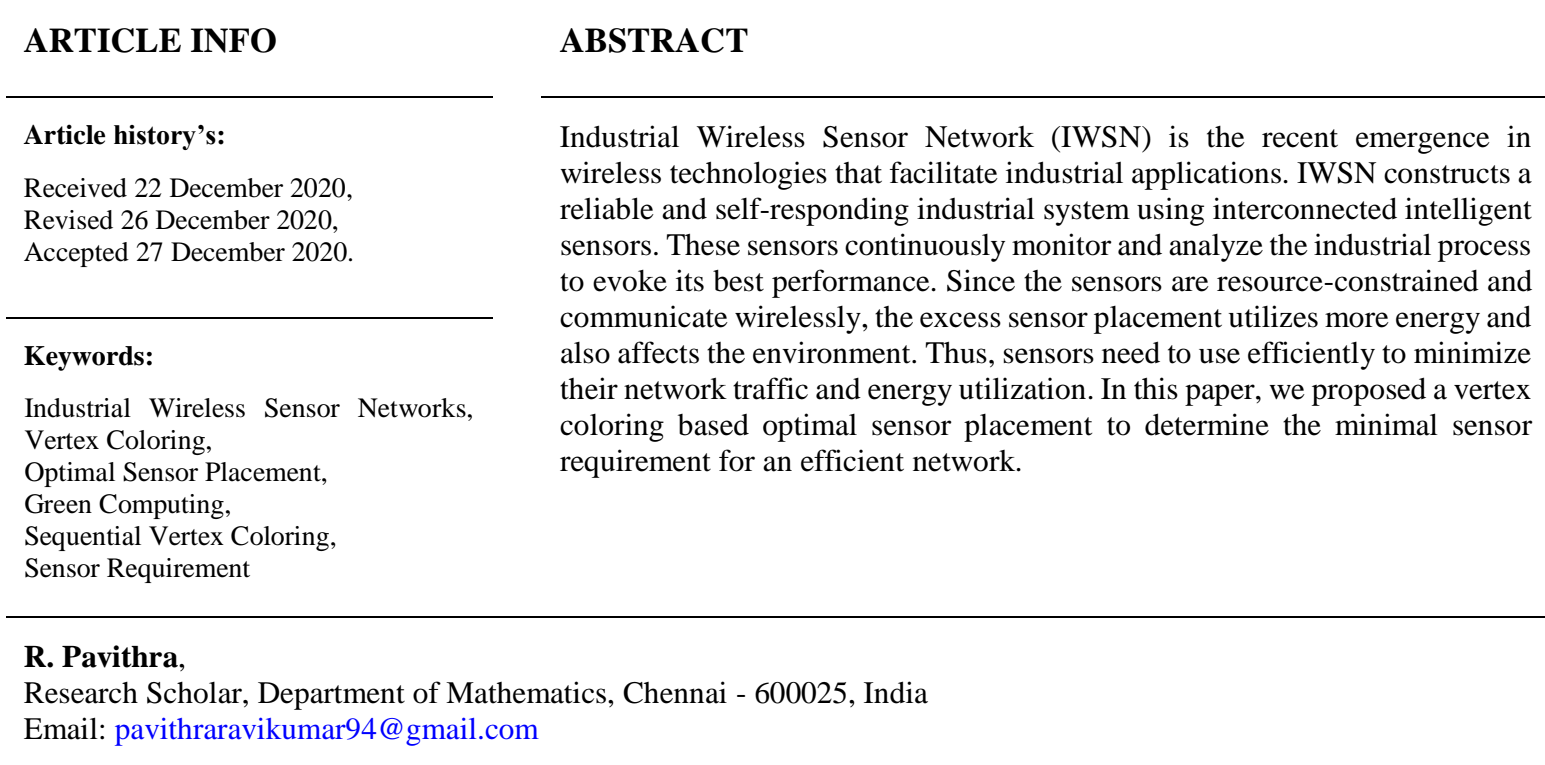

\section{INTRODUCTION}

In today's competitive industrial marketplace, due to high cost, regular maintenance, and expensive communication cables, the traditional wired industrial monitoring and control systems are evaded and started focuses on cost-effective wireless automation systems to improve the process efficiency and productivity. The cost-effective wireless automation system is feasible with the help of Wireless Sensor Networks (WSNs), and this collaboration termed as Industrial Wireless Sensor Network (IWSN). IWSN consists of a set of sensor nodes installed on industrial equipment to monitor the critical parameters such as pressure, vibration, power quality, and temperature [5]. The sensed information of each sensor is transmitted wirelessly to the base station. The base station analyses the data and provides a warning if it notifies any problem in the system. The earlier notification helps in advance replacement or repair of the equipment before its efficiency drops or fails. Thus, the cost involved in the post effects of the equipment failure can be prevented and also prohibits the cost of maintenance of the hard-wired computing system and manual monitoring.

Some of the vital IWSN applications are as follows: The rolling machines at pulp and paper mills are expensive and complicated. The minor variations in the speed, temperature, or alignment of the rollers affect the quality or operation of the machine. Hence, to monitor the devices at some specific places, sensors are deployed, such that it provides immediate notification when there are minute changes in the data. In oil refineries, maintaining the temperature of pipes is essential, but it is also tedious to do manually. Thus sensors are utilized to monitor these pipes to maintain its temperature correctly and reduce the manual work.

Similarly, accessing the real-time tank inventory data through a wired computing system leads to a delay in retrieving it. Thus tank management systems in chemical inventories use the sensor to provide instant access to the data and also to determine the value hidden within the chain. Sensors are used in the industry to detect the fire and provide early warning. Deployment of sensors provides a significant impact on protecting the lives of millions of humans. Similarly, the chemical and biological sensors play a vital role in underground mining by sensing mine disaster signals and provides early warnings to improve the safety of underground production. This processed information transmits wirelessly to enable effective communication between the surface and subsurface and analyze collected data from sensors to enhance safety measures.

Similarly, many industries utilize sensors to monitor the specified targets (machines, pipes, etc.) to improve the industrial's efficiency [22]. Therefore, the replacement of sensors for cables contributes to flexibility and cost-effectiveness. Since these sensors are resource-constrained and low cost, excess sensor 
placement preferred for efficient coverage in IWSN. Each sensor requires more power to operate appropriately in the industrial application because of the inclusive of dust, dirt, smoke, and other contaminants. On the other hand, using excess sensors in IWSN consume high power and transmits high emission for data communication and network traffic. Therefore, the efficient utilization of a significant number of sensors is a fundamental issue for green networking [10] in IWSN. This problem referred to as the optimal sensor placement for the target coverage problem, where each target in the system must monitor by at least one sensor node such that the minimum number of sensors utilized. Although optimal sensor placement for target coverage problems extensively studied in WSN, little attention required for green computing in industrial environments.

Some of the prior works concentrating on optimal sensor placement are as follows: Astorino et al. [1] formulated mixed-integer non-linear programming for target coverage problems with directional sensors. Due to its NP-hardness, the authors introduced a Lagrangian relaxation model and also proposed heuristics algorithm to find feasible solutions. Authors in [2] proposed a heuristic algorithm with two phases; the first phase ensures target coverage, and the second phase provides sensor connectivity with mobility. The simulation results ensure its better performs when compared with other techniques. Craparo et al. [3] formulated a point coverage problem as a non-linear program and an integer linear program to locate sensors for maximizing the coverage. They have also proposed to divide the best sector algorithm for identifying the optimal position for each sensor.

Guo and Jafarkhani [6] modeled sensor deployment and energy efficiency problems as a constrained source coding problem and designed the Lloyd-like algorithm to provide efficient coverage with the constraint. The simulation results describe its efficiency. Guo et al. [7] proposed a simple deterministic deployment method, which divides the targeted area into grids, and the most suitable grid is selected to deploy sensors such that it provides efficient coverage and connectivity in the network. Authors in [8] proposed a biogeographybased optimization scheme for solving the target coverage problem and a differential evolution scheme adopted to determine the optimal sensor locations for achieving k-coverage and m-connectivity in the network. An improved genetic algorithm [9] is proposed for efficient target coverage, sensor connectivity, and scheduling and also formulated the problem as a linear programming problem.

A new heuristic algorithm [11] proposed for deployment and scheduling problems in WSN. Further, the proposed algorithm performs better when compared with ant colony optimization, artificial bee colony, and particle swarm optimization techniques. In [12], the cuckoo search optimization based mobile node deployment algorithm proposed for efficient target coverage in the network. The proposed algorithm finds it a set of the best location for the sensor deployment to obtain maximum target coverage in the system.

The artificial bee colony algorithm was proposed [14] for sensor deployment under three categories of target coverage, namely, single coverage, K-coverage, and Q-coverage. Further, the deployed sensors scheduled using a heuristic algorithm for minimum energy consumption. Njoya et al. [15] proposed a multiobjective approach based genetic algorithm, referred to as a combined approach, for sensor deployment and disjoint set cover problem. The heuristic algorithm based on a genetic algorithm is proposed in [16] for target coverage in WSN to extend network lifetime. The algorithm selects the highest remaining energy nodes to cover the targets to avoid network failure and also removes redundant sensors to reduce the number of sensor nodes.

Differential evolution algorithm has proposed [17] to determine the optimal spot for the sensors in a 3D terrain. This algorithm obtained a feasible solution for the target coverage problem. Temel et al. [18] utilized the wavelet transform to get an initial set of positions for sensors. Further, to maximize its quality of coverage, a cat swarm optimization algorithm is used. Unaldi et al. [19] proposed a genetic algorithm based on wavelet transform for determining the optimal position for sensors in a 3D terrain under area coverage. It achieved maximum coverage with the minimum number of sensors when compared with the previous literature.

Wang et al. [21] proposed a novel particle swarm optimization based coverage control algorithm for energy-efficient coverage in the network. Area partition and charging discretization methods [23] proposed for sensor placement and scheduling. A mixed-integer linear programming model formulated for target coverage problems in [24] and three heuristic algorithms presented to deploy energy harvesting directional sensors in optimal spots. The result of the proposed algorithm achieves an equal success rate and solution quality. However, these deployment algorithms focused only on the effective utilization of the fixed number of sensors by determining their optimal spots. This method fails to monitor the entire targets in every scenario, because of the variation in sensor requirement due to the target's spatial coordinates, which leads to deploying excess sensors, in turn, affects the green network of IWSN. Hence, a generalized algorithm is needed, which not only optimizes the sensor position but also determines the actual requirement of sensors for the targets such that it avoids excess sensor deployment. No literature has focused on sensor requirements and its optimal spot for target coverage problem, to the best of our knowledge. Hence, Vertex Coloring based Optimal Sensor 
Placement (VC-OSP) the algorithm proposed to determine them for efficient target coverage for the green network in IWSN.

The rest of the paper organized as follows: Section 2 deals with the target coverage problem, the quality of coverage, and the implementation of the proposed algorithm for determining the sensor requirement and its optimal position. Section 3 describes the efficiency of the proposed algorithm with a series of simulation results. Section 4 provides the conclusion.

\section{PROBLEM FORMULATION}

Consider a set of $m$ targets in a fixed region $A$, as $T=\left\{T_{1}, T_{2}, \ldots, T_{m}\right\}$. Let $S=\left\{S_{1}, S_{2}, \ldots, S_{n}\right\}$ be the set of $n$ sensors ( $n$ need to be determined), where $n<m$ and $S_{R}$ be each sensor sensing range. Let $\left(x_{i}, y_{i}\right)$ and $\left(x_{j}, y_{j}\right)$ be the position of the sensor $S_{i}$ and target $T_{j}$ respectively. A target $T_{j}$ is said to be monitored by the sensor $S_{i}$ if and only if its distance between them is less than its sensing range and represented as

$$
\sqrt{\left(x_{i}-x_{j}\right)^{2}+\left(y_{i}-y_{j}\right)^{2}} \leq S_{R}
$$

where $1 \leq i \leq n, 1 \leq j \leq m$.

The coverage of the targets is represented as

$$
\lambda=\left[\lambda_{i, j}\right]
$$

where $\lambda_{i, j}=\left\{\begin{array}{c}1, S_{i} \text { monitors } T_{j} \\ 0, \text { otherwise }\end{array} ; 1 \leq i \leq n, 1 \leq j \leq m\right.$

The quality of target coverage in the network is obtained as follows

$$
T_{\text {cov }}=\sum_{j=1}^{m}\left\lceil\frac{\delta_{j}}{n}\right\rceil ; \delta_{j}=\sum_{i=1}^{n} \lambda_{i, j}
$$

This paper focuses on maximizing the quality of target coverage in the network by deploying a minimum number of sensors in the predetermined spots. Even though various algorithms have proposed to enhance the quality of coverage, the problem still needs better solutions since the existing algorithms focus on the effective utilization of a fixed number of sensors to monitor the considered targets in the network. This method fails to monitor the entire targets in every scenario because of the variation in sensor requirement due to the target's spatial coordinates, as shown in Figure 1. Four targets considered in Figure 1 and its positions are varied and described in Figure 1 (a), (b) and (c) respectively. As in every optimal sensor placement algorithm, the number of sensors is assumed as two to monitor the four targets. By optimally deploying two sensors, four targets monitored of Figure 1. (a). Whereas the same two sensors are not sufficient to provide full coverage in Figure 1 (b) and Figure 1. (c) due to the target's spatial coordinates. Thus, finding optimal spatial coordinates for a considered set of sensors provides either excess coverage or insufficient coverage depending on the target's positioning. Hence, there is a need for a generalized algorithm, which not only optimizes the sensor utilization but also determines the sensor required for the target coverage in the network. Thus, the main objective of this paper is to determine, $n$ in other words, the number of sensors required and its optimal spot such that it monitor $m$ targets. Hence, Vertex Coloring based Optimal Sensor Placement (VC-OSP) the algorithm proposed to determine them for efficient target coverage in the network.

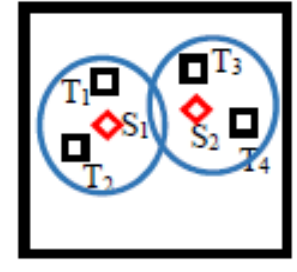

(a)

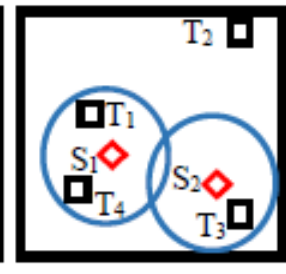

(b)

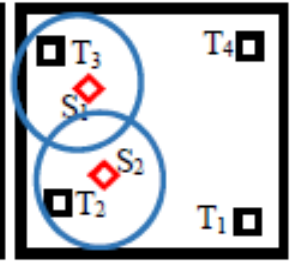

(c)

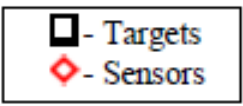

Fig. 1. Different requirement of sensors for varied target's position

\section{RESULTS AND DISCUSSION}

\subsection{The ideology of VC-OSP algorithm}

The VC-OSP algorithm proposed to determine the sensor requirement and its optimal spot to monitor all the targets in the network. As shown in Figure 1, the main reasons for the variation in the sensor requirement are the target's spatial coordinates and the sensor's sensing range. The proposed VC-OSP algorithm partitions 
the target set into the independent subsets in which each subgroup consists of a set of targets whose pairwise distance is less than the sensing range. In other words, the VC-OSP algorithm partitions the target set into sets, each group consists of a set of targets that are close to each other such that a common sensor is enough to monitor those targets. Then, the cardinality of such minimum independent subsets corresponds to the minimum number of sensors required to monitor the entire targets. Hence, to partition the targets, the VC-OSP algorithm utilizes the vertex coloring with the help of the graph, $G$. The Vertex Coloring Problem (VCP) assigns a minimum number of colors to the vertices of the graph, $G$ such that no two adjacent vertices receive the same color. From the mathematical perspective, vertex colouring partitions the vertex-set of the graph into an independent subset comprising all the vertices of the same colour. Thus, vertex coloring is simply an intuitive way to represent the target set partition.

Let us consider a sample network to analyse the process of the proposed algorithm. Considered sample network in IWSN is of size $X \times Y$ units where $X$ and $Y$ in the real-time application be $1000 \times 1000$ square meters, $2000 \times 2000$ square meters or maybe $100 \times 100$ Acers. But, to consider a simple dimension sample network for easy explanation, the size of the region $X \times Y$ units projected to $10 \times 10$ units by considering the region as $\frac{10 X}{X} \times \frac{10 Y}{Y}$ units. Let us consider a sample network of size $10 \times 10$ consist of ten targets whose coordinate points are $(3,5),(1,5),(2,4),(9,1),(4,5),(6,4),(2,10),(6,5),(10,5)$, and $(10,4)$. The sensing range of each sensor considered as three units. The objective is to determine the number of sensors required and its optimal spots to monitor these ten targets.

\subsection{Construction of undirected graph for implementation of VC-OSP algorithm}

The VC-OSP algorithm constructs an undirected graph $G=G(V(G), E(G))$ by considering targets as its vertex set and the adjacency made between the vertices (target $T_{i}$ and target $T_{j}$ ) whose distance is greater than the sensing range. The reason for this adjacency is, for any graph, $G$ in vertex coloring, the adjacent vertices receive different colors. Whereas in IWSN, the targets which are far away in other words that couldn't be monitored by the same sensor require additional sensors to monitor them. Hence, the edge set consists of the pair of targets whose distance is greater than the sensing range. Thus, each target receives different colors (sensors) using the vertex coloring technique.

The matrix representation of the adjacency of the targets is as follows

$$
a=\left[a_{i, j}\right]
$$

where $a_{i, j}=\left\{\begin{array}{l}1, \sqrt{\left(x_{i}-x_{j}\right)^{2}+\left(y_{i}-y_{j}\right)^{2}}>S_{R}, 1 \leq i \leq m, 1 \leq j \leq m \text { and }\left(x_{i}, y_{i}\right) \text { denotes the position of } \\ 0, \quad \text { otherwise }\end{array}\right.$ the target $T_{i}$

The VCP is NP-Complete because of determining the minimum number of colours required to colour all the vertices, which termed as the chromatic number, $\chi$ of a graph, $G$. But, this complex nature of VCP does not affect VC-OSP algorithm, since we aim to determine the minimum number of partitions whose cardinality, $n$ should be less than $m$ and not necessarily equal to $\chi$. To achieve $n<m$, we assume that the constructed undirected graph should not to be complete. In other words, for any considered network, we assume that there exists at least one target $T_{i}$, for any other target $T_{j}$, whose distance is less than the sensor's sensing range. Mathematically, $\exists i$, for any $j \ni a(i, j)=0$.

For the considered sample network, the adjacency matrix is obtained using equation (3) as

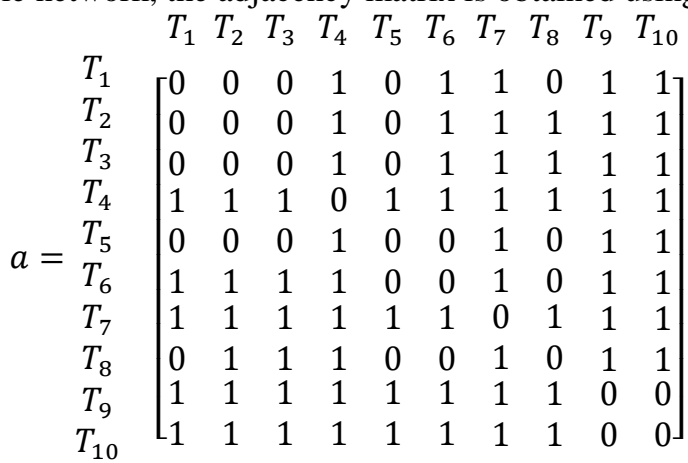

The undirected graph, $G$ of the considered sample network as the vertex set, $V(G)=\left\{T_{1}, T_{2}, T_{3}, T_{4}, T_{5}, T_{6}, T_{7}, T_{8}, T_{9}, T_{10}\right\}$ and edge set, $E(G)=\left\{\left(T_{i}, T_{j}\right): a_{i, j}=1\right\}$. Figure 2 represents the constructed undirected graph for the considered sample network. 


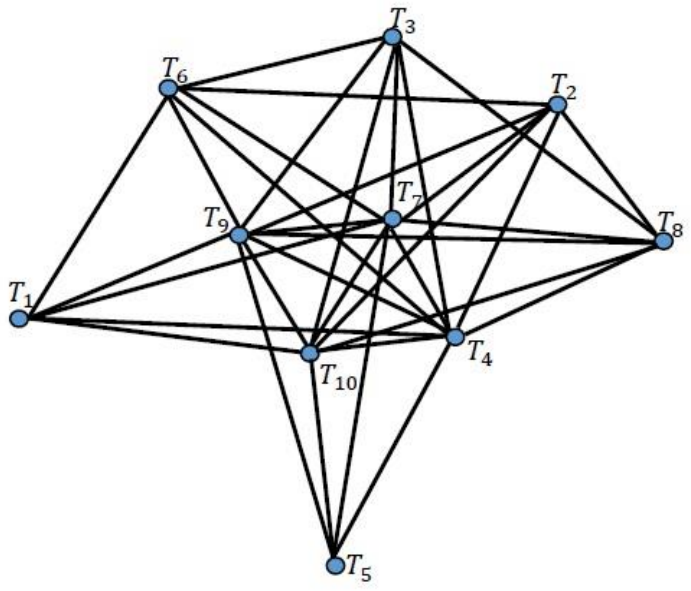

Fig. 2. The constructed undirected graph for the sample network

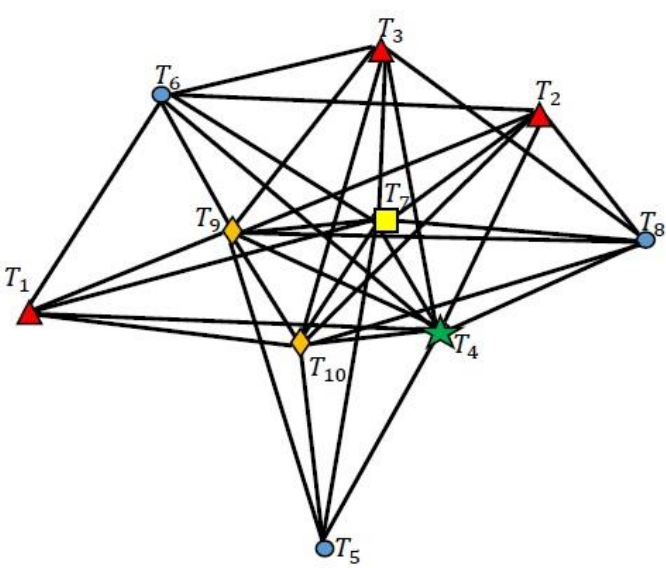

Fig. 3. Vertex coloring of the constructed graph

\subsection{Assigning colors for the constructed undirected graph using VC-OSP algorithm}

The proposed VC-OSP utilizes a sequential coloring algorithm [13] to provide vertex coloring for the constructed graph, $G$ to partition the target set. Initially, the vertex set reordered concerning its neighbour vertices, and each vertex color assigned as zero. From the reordered vertex set, the algorithm considers each vertex to determine its neighbouring vertices and colors assigned to it and represent it as neigh and cneigh respectively. This process helps to find the maximum number of colors, $m c$ utilized till previous iterations. Now, to assign color for the considered vertex, the algorithms check to reuse the colors assigned termed as availability of the colors, avail using setdiff $(1: m c, c n e i g h)$. If avail is zero, then algorithm assigns $m c+1$ color for the considered vertex otherwise assigns minimum numbered color from the avail. This process iterated until colors assigned for each vertex. Each color assigned for each vertex partitions the vertex set into independent subsets. The cardinality of those independent subsets is the number of sensors required to monitor the entire targets.

For the considered sample network, the neighbouring vertices of each vertex listed in Table 1 . The degree of the vertices mentioned in Table 1 represents the cardinality of neighbouring vertex set. The reordered vertex set concerning degree of vertices is $V(G)=\left\{T_{4}, T_{7}, T_{9}, T_{10}, T_{6}, T_{2}, T_{3}, T_{8}, T_{1}, T_{5}\right\}$. The proposed algorithm initially assigns color 'zero' for each vertex from the reordered vertex set. The sequential vertex coloring algorithm used for proper numeric coloring for the vertices and its process described in Table 2. Figure 3 shows the vertex coloring of the constructed undirected graph using the VC-OSP algorithm. From Figure 3 it is clear that the VC-OSP algorithm assigns five different colors namely red triangle, green star, blue circle, yellow square and orange diamond for the vertices $\left\{T_{1}, T_{2}, T_{3}\right\},\left\{T_{4}\right\},\left\{T_{5}, T_{6}, T_{8}\right\},\left\{T_{7}\right\},\left\{T_{9}, T_{10}\right\}$ respectively. Thus, the target set partitioned into five independent subsets with respect to its colours, and the cardinality of the independent subsets are the number of sensors required to monitor the targets. Thus, for the considered sample network, five sensors are sufficient to monitor ten targets.

Table 1. Neighboring Vertices of Each Vertex

\begin{tabular}{ccc}
\hline Vertices & Neighboring Vertices & Degree of the Vertices \\
\hline$T_{1}$ & $T_{4}, T_{6}, T_{7}, T_{9}, T_{10}$ & 5 \\
$T_{2}$ & $T_{4}, T_{6}, T_{7}, T_{8}, T_{9}, T_{10}$ & 6 \\
$T_{3}$ & $T_{4}, T_{6}, T_{7}, T_{8}, T_{9}, T_{10}$ & 6 \\
$T_{4}$ & $T_{1}, T_{2}, T_{3}, T_{5}, T_{6}, T_{7}, T_{8}, T_{9}, T_{10}$ & 9 \\
$T_{5}$ & $T_{4}, T_{7}, T_{9}, T_{10}$ & 4 \\
$T_{6}$ & $T_{1,}, T_{2}, T_{3}, T_{4}, T_{7}, T_{9}, T_{10}$ & 7 \\
$T_{7}$ & $T_{1}, T_{2}, T_{3}, T_{4}, T_{5}, T_{6}, T_{8}, T_{9}, T_{10}$ & 9 \\
$T_{8}$ & $T_{2}, T_{3}, T_{4}, T_{7}, T_{9}, T_{10}$ & 6 \\
$T_{9}$ & $T_{1}, T_{2}, T_{3}, T_{4}, T_{5}, T_{6}, T_{7}, T_{8}$ & 8 \\
$T_{10}$ & $T_{1}, T_{2}, T_{3}, T_{4}, T_{5}, T_{6}, T_{7}, T_{8}$ & 8 \\
\hline
\end{tabular}




\begin{tabular}{cccccc}
\multicolumn{7}{c}{ Table 2. Vertex Coloring Using Sequential Coloring Algorithm } \\
\hline Vertex & Neighboring vertices & $\begin{array}{c}\text { Color of } \\
\text { neighboring } \\
\text { nodes }\end{array}$ & $\begin{array}{c}\text { Maximum } \\
\text { colors used }\end{array}$ & $\begin{array}{c}\text { Available } \\
\text { color }\end{array}$ & $\begin{array}{c}\text { Color } \\
\text { assigned }\end{array}$ \\
\hline$T_{4}$ & $T_{1,}, T_{2}, T_{3}, T_{5}, T_{6}, T_{7}, T_{8}, T_{9}, T_{10}$ & 0 & 0 & - & 1 \\
$T_{7}$ & $T_{1}, T_{2}, T_{3}, T_{4}, T_{5}, T_{6}, T_{8}, T_{9}, T_{10}$ & 9 & 1 & - & 2 \\
$T_{9}$ & $T_{1}, T_{2}, T_{3}, T_{4}, T_{5}, T_{6}, T_{7}, T_{8}$ & $0,1,2$ & 2 & - & 3 \\
$T_{10}$ & $T_{1}, T_{2}, T_{3}, T_{4}, T_{5}, T_{6}, T_{7}, T_{8}$ & $0,1,2$ & 3 & 3 & 3 \\
$T_{6}$ & $T_{1}, T_{2}, T_{3}, T_{4}, T_{7}, T_{9}, T_{10}$ & $0,1,2,3$ & 3 & - & 4 \\
$T_{2}$ & $T_{4}, T_{6}, T_{7}, T_{8}, T_{9}, T_{10}$ & $0,1,2,3,4$ & 4 & - & 5 \\
$T_{3}$ & $T_{4}, T_{6}, T_{7}, T_{8}, T_{9}, T_{10}$ & $0,1,2,3,4$ & 5 & 5 & 5 \\
$T_{8}$ & $T_{2}, T_{3}, T_{4}, T_{7}, T_{9}, T_{10}$ & $1,2,3,5$ & 5 & 4 & 4 \\
$T_{1}$ & $T_{4}, T_{6}, T_{7}, T_{9}, T_{10}$ & $1,2,3,4$ & 5 & 5 & 5 \\
$T_{5}$ & $T_{4,}, T_{7}, T_{9}, T_{10}$ & $1,2,3$ & 5 & 4,5 & 4 \\
\hline
\end{tabular}

\subsection{Determining the optimal spots for the sensors}

As each independent subset requires one sensor to monitor them, the positioning of the sensor again depends on the position of the targets of each subset. The VC-OSP algorithm identifies the position of each target from each subset and determines its mean position for the optimal positioning of each sensor. In other words, the x-coordinate and y-coordinate of the sensor say $S$ is the sum of the x-coordinates and y-coordinates of each target of an independent subset divided by the cardinality of the independent set respectively, and represented as follows

$$
\bar{x}_{s}=\frac{\left(x_{1}+x_{2}+\cdots+x_{t}\right)}{t}, \bar{y}_{s}=\frac{\left(y_{1}+y_{2}+\cdots+y_{t}\right)}{t}
$$

where $1 \leq t \leq m, 1 \leq s \leq n ;\left(x_{i}, y_{i}\right)$ and $\left(\bar{x}_{l}, \bar{y}_{l}\right)$ denotes the position of the target $T_{i}$ and sensor $S_{i}$ respectively.

For the considered sample network, the optimal spot for five sensors say $S=\left\{S_{1}, S_{2}, S_{3}, S_{4}, S_{5}\right\}$ is to be determined. To determine the position of the sensor, $S_{1}$ using (5) requires the position of targets $T_{1}, T_{2}$ and $T_{3}$ which are $(3,5),(1,5)$ and $(2,4)$ respectively. Using $(5)$

$$
\bar{x}_{1}=\frac{3+1+2}{3}, \bar{y}_{1}=\frac{5+5+4}{3}
$$

The position of the sensor, $S_{1}$ is $(2,4.67)$. Similarly, the position of the sensors, $S_{2}, S_{3}, S_{4}$ and $S_{5}$ are obtained using $(5)$ are $(9,1),(5.33,4.67),(2,10)$ and $(10,4.5)$. The corresponding coverage matrix and $T_{C o v}$ using equation (2) and (3) are

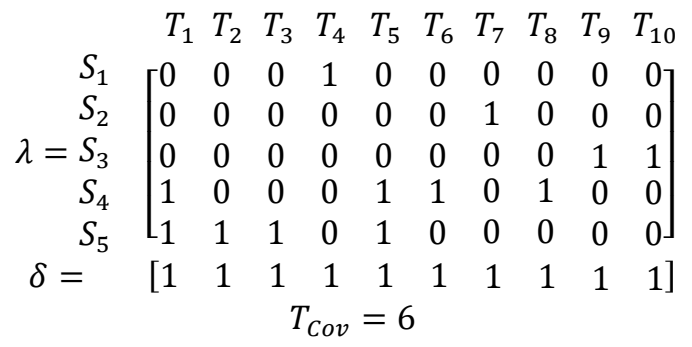

Thus, the VC-OSP algorithm determines the sensor requirement for the considered sample network as five and selected its optimal spot as $(2,4.67),(9,1),(5.33,4.67),(2,10)$ and $(10,4.5)$ such that it monitors entire targets, as shown in Fig. 4. Hence, the VC-OSP algorithm determines the sensor requirement and also determines its optimal spot for efficient target coverage in the network.

\section{PERFOMANCE EVALUATION}

The performs of the proposed VC-OSP algorithm is evaluated by a series of simulations in $1000 \times 1000$ region using MATLAB. The main idea of VC-OSP is to identify the sensor requirement and its optimal position to monitor the targets such that $T_{C O v}$ (indicates the number of targets covered by the sensors) attains maximum. 
But, the VC-OSP algorithm determines the optimal spots for sensors depending on the target's positions this sometimes leads to the deployment of sensors over the targets (Like the positions of sensor $S_{1}$ and $S_{2}$ in Figure 4. Deploying the sensors over the targets in industrial applications may increase the probability of affecting the sensors due to its pressure, vibration, temperature, etc. in the system. Hence, to provide better optimal spots for the obtained set of sensors using the VC-OSP algorithm, the algorithm utilizes the existing algorithms such as random deployment, discrete Haar Wavelet transforms algorithm [6], and cuckoo search algorithm [4]. The results obtained are tabled.

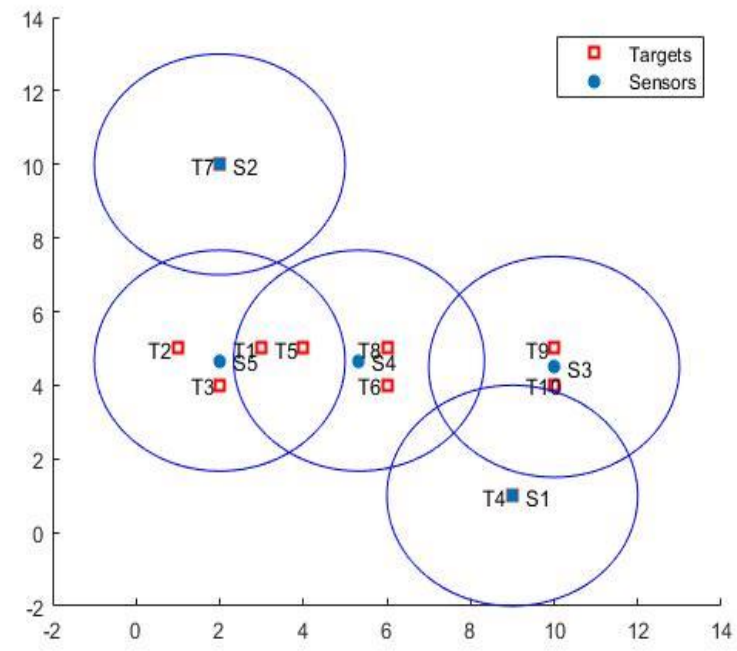

Fig. 4. The coverage of sensors using VC-OSP algorithm

Table 3 represents the estimated set of sensor requirements using VC-OSP with the variation in both targets and sensing range. The first column in Table 3 represents the variation in the targets from 100 to 250 with an increment of 25. The first row in Table 3 illustrates the variation in the sensing range from 55 to 85 , with an increment of 5. The first entry of Table 3 means that the network requires 66 sensors to monitor 100 targets using the proposed algorithm with the sensing range of 55 units. Similarly, to survey 100 targets, the system requires $60,57,56,54,51$, and 48 sensors with the sensing range of $60,65,70,75,80$, and 85 , respectively. Hence followed for other rows. Thus, Table 3 provides the sensors requirement using the VC-OSP algorithm for the considered set of targets in $1000 \times 1000$ region for the varied set of targets and sensing range.

Table 3. Number of Sensors Required for Varying Targets and Sensing Range Using VC-OSP

\begin{tabular}{llllllll}
\hline Targets & \multicolumn{7}{c}{ Sensing range } \\
\cline { 2 - 8 } & 55 & 60 & 65 & 70 & 75 & 80 & 85 \\
\hline 100 & 66 & 60 & 57 & 56 & 54 & 51 & 48 \\
125 & 74 & 70 & 68 & 63 & 62 & 57 & 54 \\
150 & 85 & 83 & 81 & 77 & 73 & 65 & 61 \\
175 & 94 & 89 & 81 & 79 & 76 & 70 & 68 \\
200 & 105 & 100 & 91 & 86 & 80 & 73 & 69 \\
225 & 116 & 107 & 101 & 95 & 87 & 83 & 79 \\
250 & 118 & 112 & 107 & 99 & 94 & 87 & 84 \\
\hline
\end{tabular}

With the help of the obtained set of sensor requirements using the VC-OSP algorithm, the optimal spots determined using evolutionary algorithms and its corresponding quality of coverage $\left(T_{C o v}\right)$ in the system also determined. Table 4, Table 5, Table 6 and Table 7 provides the estimated quality of coverage obtained under different conditions such as varying the targets set, sensing range, area, and sensor requirement respectively to evaluate the $T_{C o v}$ in the system.

Table 4 represents the $T_{\text {Cov }}$ by varying the targets from 100 to 250 targets in $1000 \times 1000$ region with the fixed sensing range of 50 units. To compare the results of random deployment, DHWT algorithm, and cuckoo search algorithm, the target positions and the number of sensors utilized should be the same. But these algorithms do not determine the sensor requirement and randomly fix the value of $n$ for target coverage. Thus, the VC-OSP algorithm utilized to determine the sensor requirement of sensors to monitor targets, and column 2 of Table 3 represents the obtained set of sensor requirements using VC-OSP. Column 3 to 5 of Table 3 shows 
the results of random deployment. Column 6 to 8 presents the results of DHWT columns 9 to 11 provide the results of the cuckoo search algorithm, and columns 12 to 14 describe the work of the VC-OSP algorithm. The minimum (Min.), average (Avg.) and maximum (Max.) $T_{\text {Cov }}$ in Table 3 are obtained by executing the algorithm fifty times. Here, the average $T_{\text {Cov }}$ of fifty experimental results is represented in percentage for easy understanding.

As depicted in Table 4, to monitor 100 targets, the sensor requirement obtained using VC-OSP is 74. By randomly deploying 74 sensors to monitor 100 targets for fifty times, the minimum $T_{C o v}$ of random deployment is obtained as 21, the average $T_{C o v}$ is $24.2 \%$ and the maximum $T_{C o v}$ is 28 . In DHWT, the minimum $T_{C o v}$ is 31 , the average $T_{C o v}$ is $37.14 \%$ and the maximum $T_{\text {Cov }}$ is 44 . By using cuckoo search algorithm the minimum $T_{\text {Cov }}$ is 56, the average $T_{C o v}$ is $59.24 \%$ and maximum $T_{C o v}$ is 59.24. Using VC-OSP algorithm the minimum, average and maximum $T_{C o v}$ is $100 \%$. Similarly, Table 4 provides the quality of coverage obtained for each varied set of targets with the determined sensor requirement using the VC-OSP algorithm. From Table 4, it is clear that the quality of coverage increases as the target set increases. The reason is, we have considered a large system $(1000 \times 1000$ region) and less set of targets (eg. 100 targets) this leads to having scattered target position in the system. Hence providing the coverage for these targets with the determined bound for sensors is comparatively less when compared with a large set of targets (e.g. 250 targets). From the results, the $T_{\text {Cov }}$ is achieved $100 \%$ using VC-OSP algorithm.

Table 4. Quality of Coverage by Varying Targets with The Sensing Range of 50 Units

\begin{tabular}{|c|c|c|c|c|c|c|c|c|c|c|c|c|c|}
\hline \multirow[t]{2}{*}{ TARGET } & \multirow{2}{*}{$\begin{array}{c}\text { SENSOR } \\
\text { USED } \\
\text { (VC-OSP) }\end{array}$} & \multicolumn{3}{|c|}{$\begin{array}{c}\text { RANDOM } \\
\text { DEPLOYMENT }\end{array}$} & \multicolumn{3}{|c|}{$\begin{array}{c}\text { DHWT } \\
\text { ALGORITHM }\end{array}$} & \multicolumn{3}{|c|}{$\begin{array}{l}\text { CUCKOO SEARCH } \\
\text { ALGORITHM }\end{array}$} & \multicolumn{3}{|c|}{$\begin{array}{c}\text { VC-OSP } \\
\text { ALGORITHM }\end{array}$} \\
\hline & & MIN. & $\begin{array}{l}\text { AVG. } \\
(\%)\end{array}$ & MAX. & MIN. & $\begin{array}{l}\text { AVG. } \\
(\%)\end{array}$ & MAX. & MIN. & $\begin{array}{l}\text { AVG. } \\
(\%)\end{array}$ & MAX. & MIN. & $\begin{array}{l}\text { AVG. } \\
(\%)\end{array}$ & MAX. \\
\hline 100 & 74 & 21 & 24.42 & 28 & 31 & 37.14 & 44 & 56 & 59.24 & 65 & 100 & 100 & 100 \\
\hline 125 & 85 & 34 & 32.24 & 45 & 44 & 43.58 & 62 & 76 & 63.21 & 84 & 125 & 100 & 125 \\
\hline 150 & 95 & 27 & 20.48 & 36 & 60 & 45.92 & 79 & 93 & 65.34 & 104 & 150 & 100 & 150 \\
\hline 175 & 104 & 39 & 25.63 & 49 & 65 & 42.86 & 85 & 113 & 67.57 & 125 & 175 & 100 & 175 \\
\hline 200 & 121 & 55 & 30.4 & 67 & 85 & 49.37 & 112 & 139 & 71.22 & 148 & 200 & 100 & 200 \\
\hline 225 & 126 & 64 & 31.68 & 75 & 97 & 49.56 & 125 & 157 & 72.72 & 168 & 225 & 100 & 225 \\
\hline 250 & 149 & 63 & 27.40 & 72 & 113 & 49.01 & 131 & 189 & 77.50 & 201 & 250 & 100 & 250 \\
\hline
\end{tabular}

Table 5 provides the estimated $T_{C o v}$ obtained by varying the sensing range, where the sensor requirement for each varied set of sensing ranges obtained using the VC-OSP algorithm to monitor 200 targets in $1000 \times$ 1000 region. Here the sensing range is varied from 55 units to 85 units. Column 2 provides the obtained sensor requirement using the VC-OSP algorithm. The results of deploying sensors to monitor 200 targets executed fifty times, the minimum, average and maximum $T_{\text {Cov }}$ are tabled in Table 4 . The average $T_{\text {Cov }}$ of random deployment, DHWT, cuckoo search, and VC-OSP algorithm are $24.25 \%, 48.87 \%, 75.91 \%$, and $100 \%$ by deploying 110 sensors with the sensing range of 55 units in $1000 \times 1000$ region respectively. Similarly, the $T_{C o v}$ is obtained and tabulated by increasing the sensing range. Here the VC-OSP algorithm performs better when compared with the random, DHWT, and cuckoo search algorithms. From the table, it is clear that as the sensing range increases, the $T_{C o v}$ also increases. The reason is as the sensing range increases the number of targets monitored by a sensor increases thus it enhances the $T_{C o v}$ in the system.

Table 5. Quality of Coverage by Varying Sensing Range to Monitor 200 Targets

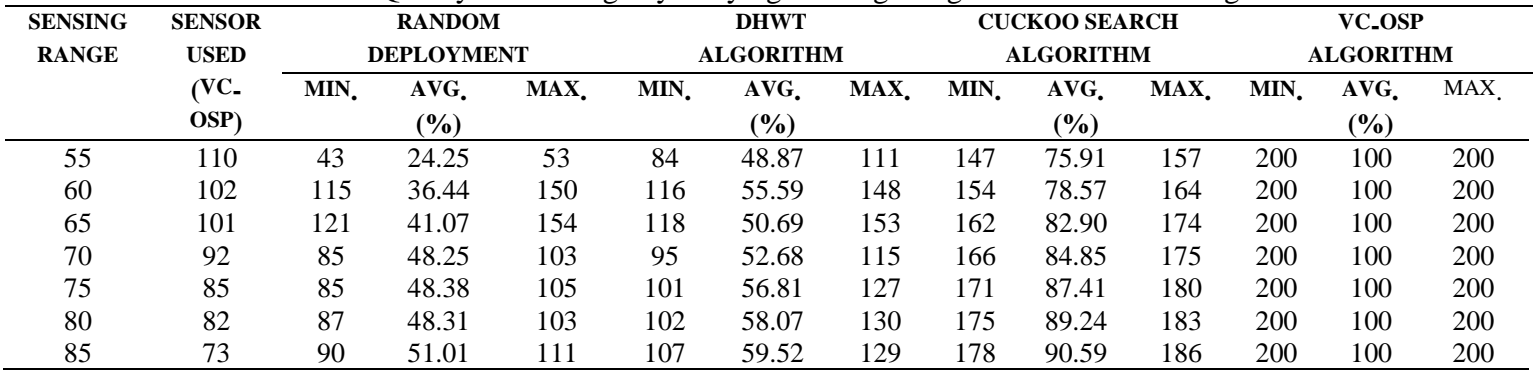

Table 6 provides the estimated $T_{\text {Cov }}$ obtained by varying the considered area from $700 \times 700$ square units to $1500 \times 1500$ square units. Column 2 of Table 6 provides the set of sensors required to monitor 150 targets with the fixed sensing range of 60 units for the various area using the VC-OSP algorithm. The results obtained by deploying these sensors categorized as the minimum, average and maximum $T_{\text {Cov }}$ by executing random, DHWT, and cuckoo search algorithms fifty times tabulated in Table 6. The average $T_{\text {Cov }}$ of random 
deployment, DHWT, cuckoo search algorithm, and VC-OSP are $75.54 \%, 76.09 \%, 88.46 \%$, and $100 \%$ by deploying 68 sensors to monitor 150 targets in $700 \times 700$ region respectively. Similarly, the $T_{\text {Cov }}$ is obtained and tabulated by increasing the considered region. From the table, it is clear that as the area increases the sensor requirement also increases while $T_{\text {Cov }}$ decreases, even though the proposed algorithm achieves $100 \%$ coverage. The reason is, monitoring the fixed targets (150 targets) in larger regions, as stated before, the position of the targets tends to scatter over the area. The spread of targets increases the sensor requirement and deploying those sensors over the broader region with the fixed sensing range leads to decrease its $T_{C o v}$.

Table 6. Quality of Coverage by Varying Area to Monitor 150 Targets

\begin{tabular}{|c|c|c|c|c|c|c|c|c|c|c|c|c|c|}
\hline \multirow[t]{2}{*}{ AREA } & \multirow{2}{*}{$\begin{array}{c}\text { SENSOR } \\
\text { USED } \\
\text { (VC- } \\
\text { OSP) }\end{array}$} & \multicolumn{3}{|c|}{$\begin{array}{c}\text { RANDOM } \\
\text { DEPLOYMENT }\end{array}$} & \multicolumn{3}{|c|}{$\begin{array}{c}\text { DHWT } \\
\text { ALGORITHM }\end{array}$} & \multicolumn{3}{|c|}{$\begin{array}{l}\text { CUCKOO SEARCH } \\
\text { ALGORITHM }\end{array}$} & \multicolumn{3}{|c|}{$\begin{array}{c}\text { VC_OSP } \\
\text { ALGORITHM }\end{array}$} \\
\hline & & MIN. & $\begin{array}{c}\text { AVG. } \\
(\%)\end{array}$ & MAX. & MIN. & $\begin{array}{c}\text { AVG. } \\
(\%)\end{array}$ & MAX. & MIN. & $\begin{array}{c}\text { AVG. } \\
(\%)\end{array}$ & MAX. & MIN. & $\begin{array}{l}\text { AVG. } \\
(\%)\end{array}$ & MAX. \\
\hline 800 & 71 & 95 & 69.60 & 116 & 88 & 66.62 & 115 & 119 & 82.48 & 131 & 150 & 100 & 150 \\
\hline 900 & 79 & 82 & 64.13 & 107 & 84 & 63.96 & 109 & 112 & 77.62 & 122 & 150 & 100 & 150 \\
\hline 1000 & 86 & 72 & 60.17 & 105 & 72 & 58.76 & 106 & 105 & 72.33 & 116 & 150 & 100 & 150 \\
\hline 1200 & 105 & 64 & 54.34 & 98 & 68 & 52.73 & 91 & 96 & 67.01 & 108 & 150 & 100 & 150 \\
\hline 1300 & 100 & 59 & 48.36 & 84 & 56 & 46.25 & 89 & 87 & 60.10 & 96 & 150 & 100 & 150 \\
\hline 1400 & 102 & 54 & 43.98 & 76 & 57 & 44.88 & 80 & 81 & 56.57 & 91 & 150 & 100 & 150 \\
\hline 1500 & 115 & 40 & 42.13 & 78 & 54 & 44.01 & 77 & 79 & 55.32 & 88 & 150 & 100 & 150 \\
\hline
\end{tabular}

For the above $T_{C o v}$ estimations, the sensor requirement $(n)$ for each experiment is determined using the VC-OSP algorithm. Thus, it is essential to check the $T_{\text {Cov }}$ by considering the set of sensors less than and more significant than the determined $n$. This estimation could help us to realize the advantage of determining the sensor requirement using the VC-OSP algorithm before optimally deployed. Hence, each algorithm executed fifty times to calculate the minimum, average and maximum $T_{C o v}$ for the sensor set less than, equal to, and more significant than the determined value $n$ in $500 \times 500$ square units.

Table 7, Figure 5 and Figure 6 provides the performance evaluation of random deployment, DHWT, and cuckoo search algorithm with the varied set of sensor requirement $(<n, n,>n)$. Column 2 of Table 6 shows the sensor requirement to monitor the mixed collection of targets in $500 \times 500$ regions with a fixed sensing range of 50 units. Using the VC-OSP algorithm, the sensor requirement to monitor 100 targets in $500 \times 500$ area with the fixed sensing range of 50 units obtained as 44 . The average $T_{\text {Cov }}$ to monitor 100 targets by randomly deploying sensors less than 44 sensors estimated as $48.38 \%$ whereas the average $T_{\text {Cov }}$ by deploying 44 sensors (estimated sensor requirement using VC-OSP algorithm) is $72.04 \%$. The result clearly shows the massive increase in $T_{C o v}$ after determining the sensor requirement. Even though the average $T_{C o v}$ of deploying sensors more excellent than 44 sensors is $72.96 \%$ (which is greater than the estimated sensor set $T_{\text {Cov }}$ ), excess sensor deployment leads to network traffic, increases the cost, and provides redundant coverage in the system. Hence, the $T_{\text {Cov }}$ obtained by deploying the estimated set of sensors (minimum sensors) using random deployment provides better coverage than the varied collection of sensor requirements and thus for the other entities. From Table 7, it is clear that determining sensor requirements before deciding its optimal spot provides a better result by enhancing the $T_{C o v}$ with minimum sensors. Similarly, the $T_{C o v}$ is estimated by varying the sensor requirement for DHWT and Cuckoo Search algorithm, and represented in Figure 5 and Figure 6, respectively.

Table 7. Quality of Coverage by Varying the Sensor Requirement for Random Deployment

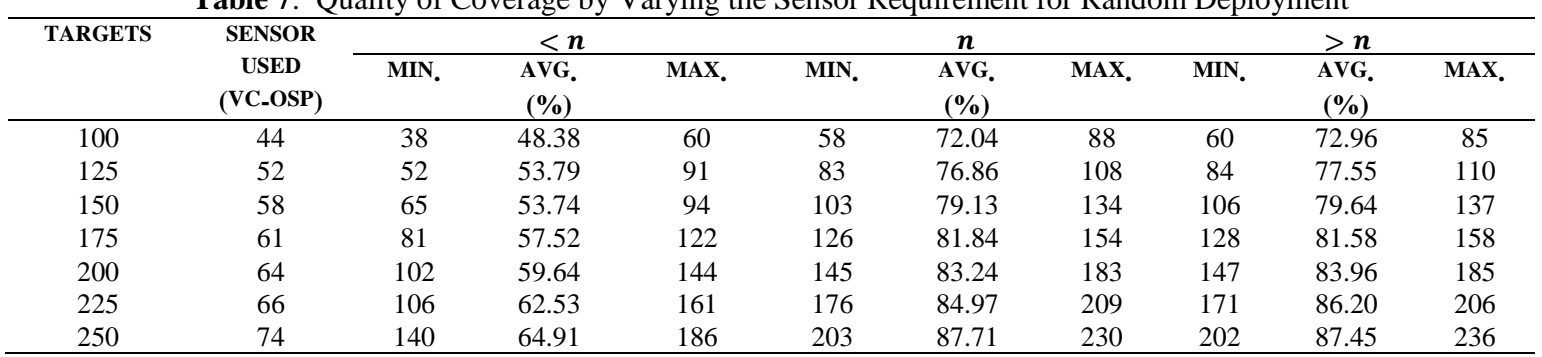




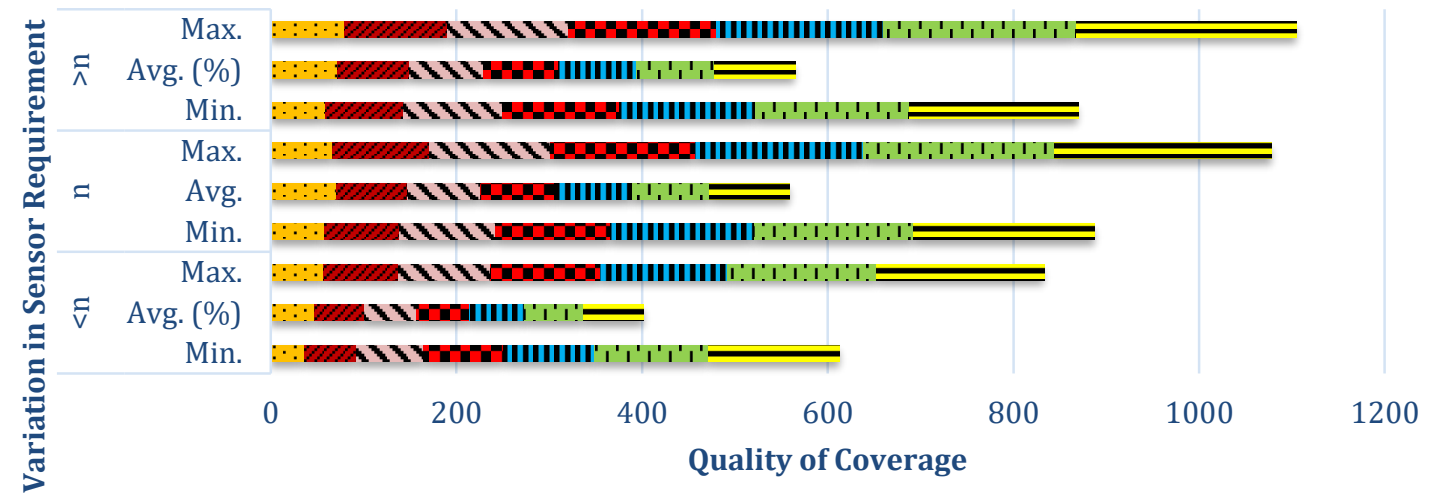

\section{Targets 125 Targets 150 Targets 175 Targets \\ II 200 Targets : 225 Targets $=250$ Targets}

Fig. 5. Quality of coverage by varying the sensor requirement for DHWT algorithm

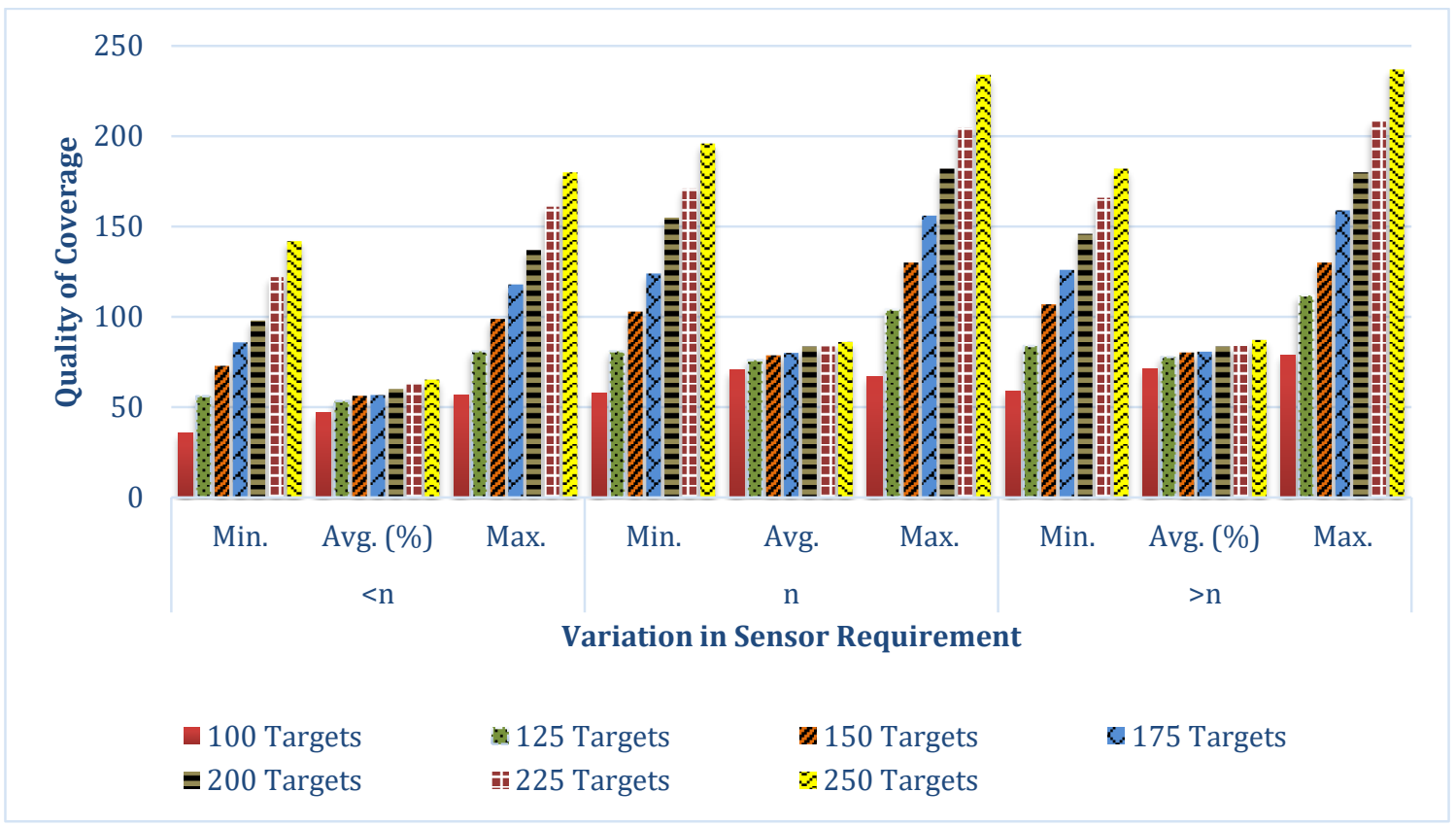

Fig. 6. Quality of coverage by varying the sensor requirement for cuckoo search algorithm

\section{CONCLUSION}

IWSNs play an enormous role in improving the productivity of industrial systems through controlling, monitoring, and maintaining the business processes. Even though IWSN is a rapidly developing area, few issues still annihilate its future exploration. The problems in IWSN are green computing, target coverage problems, network connectivity, optimal sensor placement, localization, security, and adaptability. This paper focuses on the target coverage problem by deploying the minimum number of sensors at the optimal spot for green IWSN. A simple Vertex Coloring based Optimal Sensor Placement (VC-OSP) algorithm proposed to address the above issue. The VC-OSP partitions the target set using a sequential vertex coloring algorithm to determine the sensor requirement and determines its optimal spot using the mean position of the targets in each subset. But, the VC-OSP algorithm determines the optimal spots for sensors depending on the target's parts. This method of finding positions sometimes leads to the deployment of sensors over the targets. Deploying the sensors over the targets in industrial applications may increase the probability of affecting the sensors due to its pressure, vibration, temperature, etc. in the system. Hence, to provide better optimal spots for the obtained set of sensors using the VC-OSP algorithm, the algorithm utilizes the existing evolutionary algorithms. The results obtained tabled. The simulation results clearly show that the better target coverage has achieved by each evolutionary algorithms since the sensor requirements predetermined before determining its optimal spots. 


\section{Acknowledgments}

We extend our sincere thanks to Anna centenary research fellowship and Anna University for funding our research from January 2017 to December 2019.

\section{REFERENCES}

[1] Astorino, A., Gaudioso, M., and Miglionico, G., "Lagrangian relaxation for the directional sensor coverage problem with continuous orientation", Omega, Vol. 75, pp. 77-86, 2018. DOI: https://doi.org/10.1016/j.omega.2017.03.001

[2] Choudhuri, R., and Das RK., "Coverage of targets in mobile sensor networks with restricted mobility", IEEE Access, vol. 6, pp. 10803-10813, 2018. DOI: https://doi.org/10.1109/ACCESS.2018.2801941

[3] Craparo, E. M., Fugenschuh, A., Hof, C., and Karatas, M., "Optimizing source and receiver placement in multi-static sonar networks to monitor fixed targets", European Journal of Operational Research, vol. 272, no. 3, pp. 816-831, 2019. DOI: https://doi.org/10.1016/j.ejor.2018.02.006

[4] Goyal, S., and Patterh, M. S., "Wireless sensor network localization based on cuckoo search algorithm", Wireless Personal Communications, vol. 79, no. 1, pp. 223-234, 2014. DOI: https://doi.org/10.1007/s11277-014-1850-8

[5] Gungor V. C., and Hancke, G. P., "Industrial wireless sensor networks: Challenges, design principles, and technical approaches," IEEE Transactions on industrial electronics, vol. 56, no. 10, pp. 4258-4265, 2009. DOI: https://doi.org/10.1109/TIE.2009.2015754

[6] Guo, J., and Jafarkhani, H., "Movement-efficient sensor deployment in wireless sensor network", IEEE International Conference on Communications, pp. 1-6, 2018. DOI: https://doi.org/10.1109/ICC.2018.8422124

[7] Guo, X., Zhao, C., Yang, X., and Sun, C., "A deterministic sensor node deployment method with target coverage and node connectivity," Artificial Intelligence and Computational Intelligence, vol. 7, no. 3, pp. 201-207, 2011. DOI: https://doi.org/10.1007/978-3-642-23887-1_25

[8] Gupta, G. P., and Jha, S., "Biogeography-based optimization scheme for solving the coverage and connected node placement problem for wireless sensor networks", Wireless Networks, vol. 25, no. 6, pp. 3167-3177, 2019. DOI: https://doi.org/10.1007/s11276-018-1709-0

[9] Harizan, S., and Kuila, P., "Coverage and connectivity aware energy efficient scheduling in target based wireless sensor networks: an improved genetic algorithm based approach", Wireless Networks, Vol. 25, no. 4, pp. 1995-2011, 2019. DOI: https://doi.org/10.1007/s11276-018-1792-2

[10] Huang, J., Meng, Y., Gong, X., Liu, Y., and Duan, Q., “A novel deployment scheme for green internet of things”, IEEE Internet of Things Journal, vol. 1, no. 2, pp. 196-205, 2014. DOI: https://doi.org/10.1109/JIOT.2014.2301819

[11] Krishnan, M., Rajagopal, V., and Rathinasamy, S., "Performance evaluation of sensor deployment using optimization techniques and scheduling approach for k-coverage in WSNs", Wireless Networks, vol. 24, no. 3, pp. 683-693, 2018. DOI: https://doi.org/10.1007/s11276-016-1361-5

[12] Kumari, S., and Gupta, G. P., "Cuckoo search optimization based mobile node deployment scheme for target coverage problem in underwater wireless system networks", International Conference on Intelligent Data Communication Technologies and Internet of Things, pp. 327-334, 2018. DOI: https://doi.org/10.1007/978-3-030-03146-6_36

[13] Matula D. W., Marble, G., and Isaacson, J. D., "Graph Coloring algorithms", Graph theory and computing, pp. 109-122, 1972. DOI: https://doi.org/10.1016/B978-1-4832-3187-7.50015-5

[14] Mini, S., Udgata, S., and Sabat, S., "Sensor deployment and scheduling for target coverage problem in wireless sensor networks," IEEE Sensors, Vol. 14, No. 3, pp. 636-644, 2014. DOI: https://doi.org/10.1109/JSEN.2013.2286332

[15] Njoya, A. N., Abdou, W., Dipanda, A., and Tonye, E., "Evolutionary based wireless sensor deployment for target coverage," Eleventh IEEE International Conference on Signal-Image Technology \& Internet-Based Systems, pp. 739-745, 2015. DOI: https://doi.org/10.1109/SITIS.2015.62

[16] Singh, D., Chand, S., and Kumar, B., "Genetic algorithm-based heuristic for solving target coverage problem in wireless sensor networks", Advanced computing and communication technologies, pp. 257-264, 2018. DOI: https://doi.org/10.1007/978-981-104603-2_24

[17] Sun, S., Sun, L., and Chen, S., "Research on the target coverage algorithms for 3D curved surface," Chaos Solitons Fractals, vol. 89, pp. 397-404, 2016. DOI: https://doi.org/10.1016/j.chaos.2016.02.003

[18] Temel, S., Unaldi, N., and Kaynak, O., "On deployment of wireless sensors on 3-D terrains to maximize sensing coverage by utilizing cat swarm optimization with wavelet transform," IEEE Transactions on Systems, Man, Cybernetics: System., vol. 44, no. 1,pp. 111-120, 2014. DOI: https://doi.org/10.1109/TSMCC.2013.2258336

[19] Unaldi, N., Temel, S., and Asari, V. K., "Method for optimal sensor deployment on 3D terrain utilizing a steady state genetic algorithm with a guided walk mutation operator based on the wavelet transform," Sensors, vol. 12, no. 4, pp. 5116-5133, 2012. DOI: https://doi.org/10.3390/s120405116

[20] Vijayaraju, P., Sripathy, B., Arivudainambi, D., and Balaji, S., "Hybrid memetic algorithm with two-dimensional discrete Haar wavelet transform for optimal sensor placement," IEEE Sensors, vol. 17, no. 7, pp. 2267-2278, 2017. DOI: https://doi.org/10.1109/JSEN.2017.2662951

[21] Wang, J., Ju, C., Gao, Y, Sangaiah, A. K., and Kim G. J., “A PSO based energy efficient coverage control algorithm for wireless sensor networks", Computer Mater., Vol. 56, pp. 433-446, 2018.

[22] Willig, A., " Recent and emerging topics in wireless industrial communications: A selection," IEEE Transactions on industrial informatics, vol. 4, no. 2, pp. 102-124, 2008. DOI: https://doi.org/10.1109/TII.2008.923194

[23] Wu, T., Yang, P., Dai, H., Xu, W., and Xu, M., "Charging oriented sensor placement and flexible scheduling in rechargeable WSNs", IEEE INFOCOM, pp. 73-81, 2019. DOI: https://doi.org/10.1109/INFOCOM.2019.8737502

[24] Zhu, X., Li, J., and Zhou, M., "Optimal deployment of energy harvesting directional sensor networks for target coverage", IEEE System Journal, vol. 13, no. 1, pp. 377-88, 2018. DOI: https://doi.org/10.1109/JSYST.2018.2820085 


\section{BIOGRAPHY OF AUTHORS}

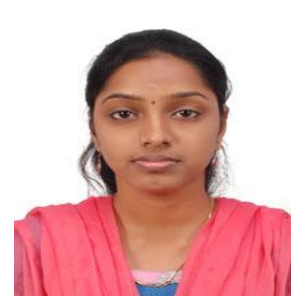

R. Pavithra received her B.Sc. degree in Mathematics from University of Madras in 2014 and M.Sc. degree in Applied Mathematics from Anna University in 2016. She is currently working towards the Ph.D. degree in Anna University, Chennai. Her research covers wavelet analysis, algorithms, graph theory and wireless sensor networks. pavithraravikumar94@gmail.com

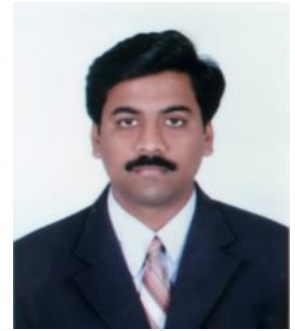

D. Arivudainambi is currently a Professor in the Department of Mathematics, Anna University, Chennai. He had been at University of Toronto, Toronto. His main research interests include network optimization, network models, queueing models with computer applications and communication systems. He is reviewer of many journals including IEEE, Elsevier, Springer and Wiley. He published many papers in reputed journals. He served as General and Technical Program Chair of numerous conferences. 2arivu@annauniv.edu 